\title{
Sexual Dimorphism of the Adult Human Retina Assessed by Optical Coherence Tomography
}

\author{
Ana Nunes - Pedro Serranho · \\ Hugo Quental · António F. Ambrósio • \\ Miguel Castelo-Branco · Rui Bernardes
}

Received: date / Accepted: date

Resumo Sexual dimorphism in the human visual system is a well-established phenomenon, and recent research has unveiled possible connections between gonadal hormones and the retina status. In the literature, the findings are quite diverse and inconclusive results have been reported as well. In the study herein, texture analysis was applied to computed optical coherence tomography (OCT) fundus images to identify differences between female and male healthy adult controls at the six neuroretinal layers. Furthermore, younger and older groups were formed to assess differences across the adult lifespan. Besides local and global texture features, the thickness of each retinal layer at study was also analysed. The vast majority of the differences between female and male groups were found from the ganglion cell layer (GCL) to the outer plexiform layer (OPL), with the retinal nerve fibre layer (RNFL) layer being the least

This study was supported by The Portuguese Foundation for Science and Technology (PEst-UID/NEU/04539/2019) and UID/04950/2017, by FEDER-COMPETE (POCI-010145-FEDER-007440 and POCI01-0145-FEDER-016428), and by Centro 2020 FEDERCOMPETE (BIGDATIMAGE, CENTRO-01-0145-FEDER-000016).

A. Nunes, H. Quental, M. Castelo-Branco and R. Bernardes

Univ Coimbra, CIBIT, ICNAS, Coimbra, Portugal

P. Serranho

Universidade Aberta, DCeT, Lisboa, Portugal

Univ Coimbra, CIBIT, Coimbra, Portugal

AF. Ambrósio

Univ Coimbra, iCBR, FMUC, Coimbra, Portugal

Univ Coimbra, CIBB, FMUC, Coimbra, Portugal

M. Castelo-Branco and R. Bernardes

Univ Coimbra, CIBIT, FMUC, Coimbra, Portugal

Corresponding author: R. Bernardes-rmbernardes@fmed.uc.pt 
distinct one. For the sub-study by age, the younger group show similar results as those for the entire population, except for the RNFL. On the other hand, the older group presents minute differences between female and male subjects. These findings suggest that studies should be well balanced by sex, and particular care should be taken in the age span of the study groups. In the present study, we also demonstrate that texture and thickness are independent, for the most part, that thickness conveys the least information, and that texture is a strong candidate biomarker of eye and central nervous system status in health and disease.

Keywords sexual dimorphism - retina - optical coherence tomography · texture analysis

\section{Introduction}

Sexual dimorphism in the human visual system is a well-established phenomenon, with recognised implications in the field of visual perception [30,27], even though sex-related differences do not seem to follow a distinct pattern [27]. The link between sex and vision in both the healthy condition and in multiple disease processes [30] suggests that sex-related differences in the visual system cannot be attributed to one single mechanism [27]. Besides, recent research regarding sexual dimorphism has unveiled possible connections between gonadal hormones and the retina status [24].

Sexual dimorphism studies focusing on the retina structure rely mostly on layer thickness measurements from optical coherence tomography (OCT) data. Across the literature, the findings are quite heterogeneous: while most studies report sex-related differences in retinal thickness [2,10,16,18, 25, 29,32], inconclusive results $[7,12]$ have been reported as well.

In recent years, new approaches to look at OCT data have been introduced, notably texture analysis [23,3]. Our group has been developing a texture analysis framework to gain further insight on the differences expressed across retinal structures, both in healthy controls [21] and in patients diagnosed with different neurodegenerative disorders [23,22]. Our methodology is based on the computation of texture features using fundus images computed from individual retinal layers.

The study herein presented constitutes an extended analysis of previous work [21], where texture analysis was applied to OCT data to distinguish between female and male healthy adult controls. In the present work, the same texture analysis methodology was applied to a larger population. Additionally, participants were split into younger and older age-groups to assess sex-related differences at different stages of the adult lifespan. Furthermore, the correlation between texture features and retinal thickness was analysed to verify if 
the texture is a surrogate for thickness or if it conveys thickness-independent information.

\section{Methods}

All processing and statistical analysis were performed using Matlab R2019b (The MathWorks Inc., Natick, MA, USA).

\subsection{Participants}

OCT data from 100 healthy controls, 50 females and 50 males, with no previous retinal pathologies, were gathered from the authors' institutional database. The data collection protocol used for gathering OCT data was approved by the Ethics Committee of the Faculty of Medicine of the University of Coimbra and performed in compliance with the Declaration of Helsinki [4]. Both eyes of each subject were analysed, except for one right eye from the female group, and one left eye from the male group, which were excluded due to scan quality, yielding a total of 198 eye scans.

Subjects were split into equal-sized groups. Furthermore, subjects were selected to ensure an exact age-match between groups, yielding identical group demographics (see table 1).

Tabela 1 Demographic data of the groups at study.

\begin{tabular}{llllll}
\hline Group & N & $\begin{array}{l}\text { Age (years) } \\
\text { Mean(STD) }\end{array}$ & $\begin{array}{l}\text { Age (years) } \\
\text { Min(Max) }\end{array}$ & $\begin{array}{l}\text { Eyes } \\
\text { Right(Left) }\end{array}$ & Total Acquisitions \\
\hline All Females & 50 & $40(17.1)$ & $19(74)$ & $49(50)$ & 99 \\
All Males & 50 & $40(17.1)$ & $19(74)$ & $50(49)$ & 99 \\
Younger Females & 25 & $27(5.6)$ & $19(39)$ & $24(25)$ & 49 \\
Younger Males & 25 & $27(5.6)$ & $19(39)$ & $25(25)$ & 50 \\
Older Females & 25 & $57(11.3)$ & $40(74)$ & $25(25)$ & 50 \\
Older Males & 25 & $57(11.3)$ & $40(74)$ & $25(24)$ & 49 \\
\hline
\end{tabular}

\subsection{OCT imaging}

The Cirrus SD-OCT 5000 (Carl Zeiss Meditec, Dublin, CA, USA) was used to gather all 198 eye scans, and the $512 \times 128$ Macular Cube protocol was used at the same retinal position (centred on the macula) for all OCT acquisitions. 


\subsection{Image processing}

OCT data were processed using the OCT Explorer software (Retinal Image Analysis Lab, Iowa Institute for Biomedical Imaging, Iowa City, IA, USA) [19, $11,1]$ to segment the six innermost layers of the retina: the retinal nerve fibre layer (RNFL), the ganglion cell layer (GCL), the inner plexiform layer (IPL), the inner nuclear layer (INL), the outer plexiform layer (OPL) and the outer nuclear layer (ONL).

For each of the six layers at study, a mean value fundus (MVF) image [13] was computed in which each pixel is the average of the corresponding A-scan zeroing all A-scan values outside the layer of interest. As both eyes from each subject were considered for the present analysis, all left-eye MVF images were horizontally flipped to match the temporal and nasal regions across all eye scans, and the computed texture metrics to present the same relative position across eyes. For a visual example of a MVF image for each of the six layers, please refer to figure 1.

Figura 1 Colour-coded MVF images from the right eye of a healthy control [23]. From left to right and top to bottom: RNFL, GCL, IPL, INL, OPL and ONL layer fundus images.

\subsection{Texture analysis}

Texture features were computed for each MVF image, following an approach used previously [23, 21,22,20]. Two different texture analysis methods were applied to MVF images: the grey-level co-occurrence matrix (GLCM) [15] and the dual-tree complex wavelet transform (DTCWT) [26]. Using the GLCM, local intensity variations can be highlighted by iteratively examining pixel pairs. On the other hand, the DTCWT examines spatial frequencies at multiple scales, thus enabling the identification of coarser texture properties [17]. For these reasons, the GLCM was used to capture localised grey-level variations, while the DTCWT was applied to assess the texture profile of the macular region on a larger scale.

Regarding the GLCM, each image was first down-sampled to $128 \times 128$ pixels to obtain isotropic sampling in the horizontal and vertical directions and converted to 16 grey-level to limit the size of the GLCM matrix. Images were then split into $7 \times 7$ blocks, which were independently analysed, to obtain the local GLCM-based features. Please refer to figure 2 for a visual scheme of this division into blocks. In this 49 -block grid, the central row ( $4^{\text {th }}$ row) and column $\left(4^{\text {th }}\right.$ column) were not considered in the analysis to exclude the foveal region, where the six layers at study are significantly reduced (if not inexistent). For 
each block, four GLCMs were computed, corresponding to four different pixel pair orientations (i.e. relative positions of any two pixels under analysis): $0^{\circ}$, $45^{\circ}, 90^{\circ}$, and $135^{\circ}$. Also, the distance of one pixel $(d=1)$ was considered and $180^{\circ}$ apart angles were considered the same, e.g. the pixel-pairs $(i, j)(i, j+d)$ and $(i, j)(i, j-d)$, corresponding respectively to the angles $0^{\circ}$ and $180^{\circ}$, contribute to the same angle $\left(0^{\circ}\right)$. For each block, 20 features were computed for each of the four orientations: a) Autocorrelation, b) Cluster Prominence, c) Cluster Shade, d) Correlation, e) Difference Entropy, f) Difference Variance, g) Dissimilarity, h) Entropy, i) Homogeneity (also known as Inverse Difference Moment), j) Inertia (also known as Contrast), k) Information Measure of Correlation 1 (IMC1), l) Information Measure of Correlation 2 (IMC2), m) Inverse Difference Moment Normalized (IDN), n) Inverse Difference Normalized (INN), o) Maximum Probability, p) Sum Average, q) Sum Entropy, r) Sum of Squares, s) Sum Variance, and t) Uniformity (also known as Angular Second Moment, or Energy). The definition of texture features a) and o) can be found in [14], features b) and c) in [9], feature g) in [28], features) $m$ and $\mathrm{n}$ ) in [8] and the remaining 13 features in [15]. The maximum value across the four orientations was selected as the ultimate feature value for each block to accommodate differences in orientation such as for the nerve fibres. Finally, blocks were aggregated into quadrants (composed of $3 \times 3$ blocks each - Fig. 2 ): the temporal-superior (Q1), nasal-superior (Q2), temporal-inferior (Q3) and nasal-inferior (Q4) quadrants. The average of each texture feature, across the $3 \times 3$ blocks, was used as the final feature value for the quadrant.

Figura 2 Computed fundus image from the volumetric macular cube scan of the right eye of a healthy control subject [23]. Each of the $7 \times 7$ blocks show the individually analysed areas which results were later aggregated into larger regions (shaded areas). Image axes are: $\mathrm{x}$-axis (horizontal) - temporal (left) to nasal (right) and y-axis (vertical) superior (top) to inferior (bottom).

The DTCWT, on the other hand, was applied to the full macular area covered by the MVF image (i.e. no downsampling, and no division of the image area into quadrants). The variance of the magnitude of the DTCWT complex coefficients was computed for six directionally selective image subbands $\left( \pm 15^{\circ}, \pm 45^{\circ}\right.$ and $\left.\pm 75^{\circ}\right)$, following the approach described in [6,31]. These six directional variance values were used as the global DTCWT-based features for each MVF image.

In total, 86 texture features were computed for each of the six retinal layers at study, $80(20 \times 4$ quadrants $)$ local GLCM-based and six global DTCWTbased features. 
2.5 Thickness measurements

Five thickness values were computed per retinal layer, one considering the average thickness for the entire image area, and four average thickness values one per retinal quadrant considering only the area used for the GLCM texture values (Fig. 2).

\subsection{Statistical analysis}

All texture features and thickness measurement values were tested for normality using the Kolmogorov-Smirnov test, with a $10 \%$ significance level for a more conservative decision regarding the normality of the distributions. For each, only when both groups follow a normal distribution, the two-sample t-test was used to test mean differences between sex groups. Otherwise, the Mann-Whitney U-test (non-parametric) was used instead. The obtained $p$ values for the mean difference test were separated into three significance levels: $p \leq 0.05, p \leq 0.01$ and $p \leq 0.001$.

The correlations between texture features and thickness values were computed using the Pearson correlation coefficient. GLCM texture features for each quadrant were correlated with the respective quadrant thickness value, and DTCWT texture features were correlated with the mean thickness value for the entire imaged area.

\section{Results}

The first analysis concerns all females and all males in the present study. Table 2 shows the list of texture features and thickness measurements presenting statistically significant differences between groups.

When comparing these two groups, one can find that the majority of the differences are present within the GCL to the OPL layers. In contrast, the ONL and the RNFL only present, respectively, five and two features at a significance level below $1 \%$.

While features presenting statistically significant differences are mostly spread in the macular region in the INL, across three quadrants, they are mostly present in two quadrants in the GCL and the IPL, and in one quadrant in the OPL. Highest statistical differences, for GLCM texture features, can be found mostly in the GCL and, to a more considerable extent, in the IPL. Q3, the superior-nasal quadrant, holds consistent differences at consecutive layers of the retina - from the GCL to the INL. As for the global features (DTCWT), these present very high statistically significant differences at the GCL and INL only. 
Conversely, statistically significant differences for thickness measurements are merely residual. These were found only in the IPL and INL layers and only in two quadrants each.

The secondary analysis in the present study aims to clarify if above-found differences are kept during the lifespan or modify with age. Hence, the female and male groups were split into younger and older age-groups and the above analysis repeated. Tables 3 and 4 present the results for, respectively, the younger and older groups.

The fundamental difference is the much fewer differences between females and males in the older group, while the differences in the younger group overlap to a large extent those found for the entire population at study (table 2).

The main findings for the younger group (table 3) are the significant increase in differences found at the RNFL and the further spread across the macular region of the differences found in the INL. Similarly to findings for the entire population at study, Q3 presents consistent differences from the GCL to the INL.

On the other hand, table 4 shows that for older subjects the differences are fewer than those found for the entire population and mostly at the standard significant level, except for the differences found in the IPL in Q1.

Finally, the correlations found between texture features and thickness values show that textures metrics are not a surrogate for thickness measurements, and convey important information on the status of the retina not conveyed by thickness alone. From table 5, only three texture metrics, all of the global type (DTCWT), present a strong correlation (absolute value over 0.7) with thickness for the same area and layer. All other 17 features present only moderate Pearson correlations with thickness, meaning that the majority of the features are independent of thickness as measured by the Pearson correlation coefficient.

\section{Discussion}

The correlation between sex and retinal diseases are known [24] and were put forward as a consequence of gonadal hormones. Not only this correlation may have an impact on the use of hormone therapy in the treatment of retinal disorders [24], but it may also impact the assessment of neurological disorders based on retinal imaging. The unbalanced number of subjects, from the viewpoint of study subjects' sex, on the one hand, and the spread of age, on the other hand, may mask potential findings in these studies. Furthermore, while the majority of studies rely on retinal thickness measurements, the present 
Tabela 2 Texture features and thickness measurements presenting statistically significant differences between all the female and male subjects for the retinal nerve fibre layer (RNFL); ganglion cell layer (GCL); inner plexiform layer (IPL); inner nuclear layer (INL); outer plexiform layer (OPL), and; outer nuclear layer (ONL). Hyphens (-) represent a $p$-value $>$ 0.05 (non-significant difference), the green-coloured circles $(-)$ represent a $p$-value $\leq 0.05$, the orange-coloured squares $(\square)$ represent a $p$-value $\leq 0.01$ and the red-coloured asterisks (*) represent a $p$-value $\leq 0.001$. Q1 - Superior-temporal quadrant, Q2 - Superior-nasal quadrant, Q3 - Inferior-temporal quadrant and Q4 - Inferior-nasal quadrant. IDN stands for Inverse Difference Moment Normalized; in the features IMC1 and IMC2, IMC stands for Information Measure of Correlation; and INN stands for Inverse Difference Normalized. Thickness values are presented by quadrant and globally, so they can be directly compared to the respective local/global texture features.

\begin{tabular}{|c|c|c|c|c|c|c|c|c|c|c|c|}
\hline Layer & Parameter & Q1 & Q2 & Q3 & Q4 & Layer & Parameter & Q1 & Q2 & Q3 & Q4 \\
\hline \multirow{23}{*}{ RNFL } & Cluster Shade & $\square$ & - & - & - & \multirow{23}{*}{ GCL } & Autocorrelation & 0 & - & $\square$ & - \\
\hline & Sum Average & $\square$ & - & - & - & & Cluster Prominence & 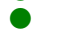 & - & $\square$ & - \\
\hline & Sum of Squares & 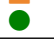 & - & - & - & & Correlation & - & - & * & - \\
\hline & Variance $75^{+}$(Global) & & & & & & Difference Entropy & - & - & $\square$ & - \\
\hline & Variance $75^{-}$(Global) & & & & & & Difference Variance & - & - & $\square$ & - \\
\hline & & & & & & & Dissimilarity & - & - & 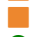 & - \\
\hline & & & & & & & Entropy & - & - & 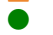 & - \\
\hline & & & & & & & Homogeneity & 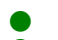 & - & * & - \\
\hline & & & & & & & IDN & 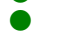 & - & * & - \\
\hline & & & & & & & IMC1 & - & - & 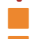 & - \\
\hline & & & & & & & IMC2 & - & - & $\square$ & - \\
\hline & & & & & & & Inertia & - & - & $\square$ & - \\
\hline & & & & & & & INN & - & - & * & - \\
\hline & & & & & & & Maximum Probability & - & - & * & - \\
\hline & & & & & & & Sum Entropy & 0 & - & ? & O \\
\hline & & & & & & & Sum Variance & 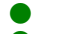 & - & 0 & - \\
\hline & & & & & & & Uniformity & 0 & - & 口 & 0 \\
\hline & & & & & & & Variance $15^{+}$(Global) & & & & \\
\hline & & & & & & & Variance $15^{-}$(Global) & & & & \\
\hline & & & & & & & Variance $45^{+}$(Global) & & & & \\
\hline & & & & & & & Variance $45^{-}$(Global) & & & & \\
\hline & & & & & & & Variance $75^{+}$(Global) & & & & \\
\hline & & & & & & & Variance $75^{-}$(Global) & & & & \\
\hline \multirow{25}{*}{ IPL } & Autocorrelation & $\square$ & - & * & - & \multirow{25}{*}{ INL } & Autocorrelation & - & - & O & - \\
\hline & Cluster Prominence & $\square$ & - & * & - & & Cluster Prominence & $\square$ & $\square$ & 0 & - \\
\hline & Correlation & 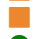 & - & * & - & & Correlation & $\square$ & $\square$ & 8 & - \\
\hline & Difference Entropy & 0 & - & * & - & & Difference Entropy & $\square$ & 0 & 0 & - \\
\hline & Difference Variance & 0 & - & 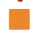 & - & & Difference Variance & $\square$ & 0 & 0 & - \\
\hline & Dissimilarity & 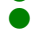 & - & $\square$ & - & & Dissimilarity & $\square$ & 0 & 0 & - \\
\hline & Entropy & - & - & * & - & & Entropy & 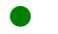 & - & - & - \\
\hline & Homogeneity & $\square$ & - & * & - & & Homogeneity & $\square$ & $\square$ & O & - \\
\hline & IDN & 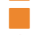 & - & * & - & & IDN & 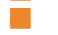 & $\overline{-}$ & 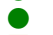 & - \\
\hline & IMC1 & * & - & - & - & & IMC1 & 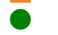 & 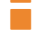 & 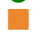 & - \\
\hline & IMC2 & 0 & - & * & - & & IMC2 & 口 & 口 & 0 & - \\
\hline & Inertia & 0 & - & 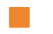 & - & & Inertia & $\square$ & 0 & 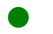 & - \\
\hline & INN & 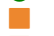 & - & * & - & & INN & $\square$ & 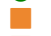 & 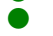 & - \\
\hline & Maximum Probability & 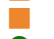 & - & 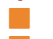 & - & & Maximum Probability & 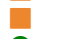 & $\square$ & 0 & - \\
\hline & Sum Entropy & 0 & - & $\square$ & - & & Sum Average & 0 & - & - & - \\
\hline & Sum Variance & 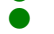 & - & $\square$ & - & & Sum Entropy & 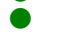 & 0 & - & - \\
\hline & Uniformity & $\square$ & - & $*$ & - & & Uniformity & $\square$ & $\square$ & 0 & - \\
\hline & Variance $45^{+}$(Global) & & & & & & Variance $15^{+}$(Global) & & & & \\
\hline & Variance $45^{-}$(Global) & & & & & & Variance $15^{+}$(Global) & & & & \\
\hline & Variance $75^{-}$(Global) & & & & & & Variance $15^{-}$(Global) & & & & \\
\hline & \multirow[t]{5}{*}{ Thickness (Quadrants) } & \multirow[t]{5}{*}{$\square$} & \multirow[t]{5}{*}{-} & \multirow[t]{5}{*}{ O } & \multirow[t]{5}{*}{-} & & Variance $45^{+}$(Global) & & & & \\
\hline & & & & & & & Variance $45^{-}$(Global) & & & & \\
\hline & & & & & & & Variance $75^{+}$(Global) & & & & \\
\hline & & & & & & & Thickness (Quadrants) & $\mathcal{U}$ & - & - & - \\
\hline & & & & & & & Thickness (Global) & & & & \\
\hline \multirow{17}{*}{ OPL } & Autocorrelation & - & $\square$ & O & - & & Cluster Shade & - & - & O & - \\
\hline & Cluster Prominence & - & 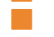 & - & - & & Correlation & - & - & - & 0 \\
\hline & Correlation & - & 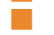 & - & - & & Difference Entropy & - & 0 & - & 0 \\
\hline & Difference Entropy & - & $\bar{\square}$ & - & - & & Dissimilarity & - & 0 & - & - \\
\hline & Difference Variance & - & $\square$ & - & - & & Homogeneity & - & - & - & O \\
\hline & Dissimilarity & - & $\square$ & - & - & & IDN & - & - & - & 0 \\
\hline & Entropy & - & $\overline{-}$ & - & - & & INN & - & - & - & 0 \\
\hline & Homogeneity & - & ㅁ & - & - & & Sum Average & - & - & - & * \\
\hline & IDN & - & $\bar{\square}$ & - & - & ONL & Sum of Squares & - & - & - & * \\
\hline & IMC1 & - & $\square$ & - & - & & Sum Variance & - & - & - & 0 \\
\hline & IMC2 & - & $\square$ & - & - & & Variance $45^{+}$(Global) & & & & \\
\hline & Inertia & - & $\bar{\square}$ & - & - & & Variance $45^{-}$(Global) & & & & \\
\hline & INN & - & 口 & - & - & & Variance $75^{+}$(Global) & & & & \\
\hline & Maximum Probability & - & 9 & 0 & - & & Variance $75^{-}$(Global) & & & & \\
\hline & Sum Entropy & - & 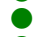 & - & - & & & & & & \\
\hline & Sum Variance & - & ? & O & - & & & & & & \\
\hline & Uniformity & - & 口 & - & - & & & & & & \\
\hline
\end{tabular}


Tabela 3 Texture features and thickness measurements presenting statistically significant differences between the younger female and male groups for the same layers. The notation is as in table 2 .

\begin{tabular}{|c|c|c|c|c|c|c|c|c|c|c|c|}
\hline Layer & Parameter & Q1 & Q2 & Q3 & Q4 & Layer & Parameter & Q1 & Q2 & Q3 & $\mathrm{Q} 4$ \\
\hline \multirow{25}{*}{ RNFL } & Autocorrelation & - & 0 & - & - & \multirow{25}{*}{ GCL } & Autocorrelation & - & - & 0 & - \\
\hline & Cluster Prominence & $\square$ & - & - & - & & Cluster Prominence & - & - & 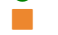 & - \\
\hline & Cluster Shade & 0 & - & - & 0 & & Correlation & - & - & 口 & - \\
\hline & Correlation & 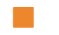 & $\square$ & - & - & & Difference Entropy & - & - & $\square$ & - \\
\hline & Difference Entropy & 0 & 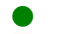 & - & - & & Difference Variance & - & - & $\square$ & - \\
\hline & Difference Variance & 0 & 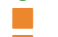 & - & - & & Dissimilarity & - & - & $\bar{\square}$ & - \\
\hline & Dissimilarity & 0 & - & - & - & & Entropy & - & - & 0 & - \\
\hline & Entropy & 0 & 8 & - & - & & Homogeneity & - & - & 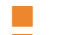 & - \\
\hline & Homogeneity & 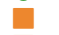 & 0 & - & 0 & & IDN & - & - & $\overline{-}$ & - \\
\hline & IDN & $\square$ & 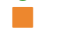 & - & - & & IMC1 & - & - & $\overline{-}$ & - \\
\hline & IMC1 & 0 & 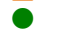 & - & - & & IMC2 & - & - & 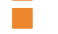 & - \\
\hline & $\mathrm{IMC} 2$ & 0 & 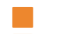 & - & - & & Inertia & - & - & 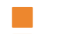 & - \\
\hline & Inertia & 0 & 口 & - & - & & INN & - & - & 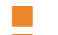 & - \\
\hline & INN & 口 & 0 & - & 0 & & Maximum Probability & - & - & $\square$ & - \\
\hline & Maximum Probability & 0 & 口 & - & - & & Sum Entropy & - & - & O & - \\
\hline & Sum Average & $\square$ & - & - & - & & Sum of Squares & - & - & - & 0 \\
\hline & Sum Entropy & 0 & O & - & - & & Sum Variance & - & - & O & - \\
\hline & Sum Variance & - & 0 & - & - & & Uniformity & - & - & 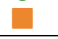 & - \\
\hline & Uniformity & 口 & - & - & - & & Variance $15^{+}$(Global) & & & & \\
\hline & Variance $45^{+}$(Global) & & & & & & Variance $15^{-}$(Global) & & & & \\
\hline & Variance $75^{+}$(Global) & & & & & & Variance $45^{+}$(Global) & & & & \\
\hline & Variance $75^{-}$(Global) & & & & & & Variance $45^{-}$(Global) & & & & \\
\hline & \multirow[t]{3}{*}{ Thickness (Quadrants) } & \multirow[t]{3}{*}{-} & \multirow[t]{3}{*}{-} & \multirow[t]{3}{*}{-} & \multirow[t]{3}{*}{ O } & & Variance $75^{+}$(Global) & & & & \\
\hline & & & & & & & Variance $75^{-}$(Global) & & & & \\
\hline & & & & & & & Thickness (Quadrants) & - & 0 & - & - \\
\hline \multirow{25}{*}{ IPL } & Autocorrelation & - & - & * & - & \multirow{25}{*}{ INL } & Autocorrelation & - & - & 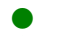 & - \\
\hline & Cluster Prominence & - & - & 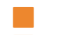 & - & & Cluster Prominence & 0 & $\square$ & 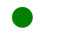 & 0 \\
\hline & Correlation & - & - & 口 & - & & Cluster Shade & - & 0 & - & - \\
\hline & Difference Entropy & - & - & 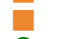 & - & & Correlation & 0 & 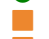 & 0 & 0 \\
\hline & Difference Variance & - & - & 0 & - & & Difference Entropy & 8 & 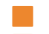 & 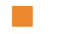 & - \\
\hline & Dissimilarity & - & - & 口 & - & & Difference Variance & 0 & $\square$ & $\square$ & - \\
\hline & Entropy & - & - & * & - & & Dissimilarity & 0 & $\overline{-}$ & 0 & - \\
\hline & Homogeneity & - & - & * & - & & Entropy & - & - & 0 & 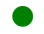 \\
\hline & IDN & - & - & 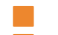 & - & & Homogeneity & 0 & 0 & 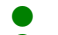 & - \\
\hline & IMC2 & - & - & $\square$ & - & & IDN & 0 & $\square$ & 0 & 0 \\
\hline & IMC1 & - & - & 口 & - & & IMC1 & 0 & $\square$ & O & - \\
\hline & Inertia & - & - & ○ & - & & IMC2 & 0 & 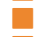 & 0 & 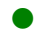 \\
\hline & INN & - & - & 口 & - & & Inertia & 0 & 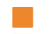 & 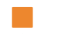 & - \\
\hline & Maximum Probability & - & - & - & - & & INN & 0 & 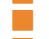 & 0 & - \\
\hline & Sum Entropy & - & - & $\bar{\square}$ & - & & Maximum Probability & 0 & $\square$ & 0 & 0 \\
\hline & Sum Variance & - & - & $\square$ & - & & Sum Entropy & - & 0 & 0 & - \\
\hline & Uniformity & - & - & * & - & & Sum Variance & - & - & 0 & - \\
\hline & Thickness (Quadrants) & 0 & - & - & - & & Uniformity & 0 & 0 & 0 & - \\
\hline & & & & & & & Variance $15^{+}$(Global) & & & & \\
\hline & & & & & & & Variance $15^{-}$(Global) & & & & \\
\hline & & & & & & & Variance $45^{+}$(Global) & & & & \\
\hline & & & & & & & Variance $45^{-}$(Global) & & & & \\
\hline & & & & & & & Variance $75^{+}$(Global) & & & & \\
\hline & & & & & & & Variance $75^{-}$(Global) & & & & \\
\hline & & & & & & & Thickness (Global) & & & & \\
\hline & Autocorrelation & - & $\square$ & - & - & & Cluster Shade & 0 & 0 & - & - \\
\hline & Cluster Prominence & - & 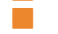 & - & 0 & & Correlation & - & - & - & 0 \\
\hline & Correlation & - & 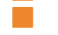 & - & 0 & & Difference Entropy & - & 0 & - & 0 \\
\hline & Difference Entropy & - & 口 & - & 0 & & Difference Variance & - & - & - & 0 \\
\hline & Difference Variance & - & $\square$ & - & - & & Dissimilarity & - & 0 & - & - \\
\hline & Dissimilarity & - & 口 & - & - & & Homogeneity & - & - & - & 0 \\
\hline & Entropy & - & 口 & - & - & & IDN & - & - & - & - \\
\hline & Homogeneity & - & 口 & - & 0 & & IMC1 & - & 0 & - & - \\
\hline & IDN & - & 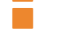 & - & 0 & & Inertia & - & - & - & 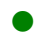 \\
\hline OPL & IMC1 & - & 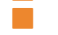 & - & - & ONL & INN & - & - & - & 0 \\
\hline & $\mathrm{IMC} 2$ & - & $\square$ & - & 0 & & Sum of Squares & - & - & 0 & - \\
\hline & Inertia & - & 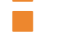 & - & 0 & & Variance $45^{-}$(Global) & & & & \\
\hline & INN & - & $\bar{\square}$ & - & 0 & & Variance $75^{+}$(Global) & & & & \\
\hline & Maximum Probability & - & $\overline{-}$ & - & - & & Variance $75^{-}$(Global) & & & & \\
\hline & Sum Entropy & - & 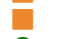 & - & - & & & & & & \\
\hline & Sum Variance & - & 0 & - & - & & & & & & \\
\hline & Uniformity & - & 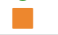 & - & 0 & & & & & & \\
\hline & Thickness (Quadrants) & 0 & - & - & - & & & & & & \\
\hline
\end{tabular}


Tabela 4 Texture features and thickness measurements presenting statistically significant differences between the older female and male groups for the same layers. The notation is as in table 2 .

\begin{tabular}{|c|c|c|c|c|c|}
\hline Layer & Parameter & Q1 & Q2 & Q3 & Q4 \\
\hline RNFL & Thickness (Quadrants) & - & - & 0 & - \\
\hline \multirow{4}{*}{ GCL } & Maximum Probability & - & - & 0 & - \\
\hline & Variance $15^{+}$(Global) & & & & \\
\hline & Variance $15^{-}$(Global) & & & & \\
\hline & Variance $45^{-}$(Global) & & & & \\
\hline \multirow{20}{*}{ IPL } & Autocorrelation & 0 & - & - & - \\
\hline & Cluster Prominence & 口 & - & - & - \\
\hline & Correlation & $\bar{\square}$ & - & 0 & - \\
\hline & Difference Entropy & $\square$ & - & - & - \\
\hline & Difference Variance & 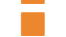 & - & - & - \\
\hline & Dissimilarity & $\bar{\square}$ & - & - & - \\
\hline & Entropy & 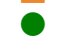 & - & - & - \\
\hline & Homogeneity & 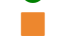 & - & - & - \\
\hline & IDN & $\square$ & - & ○ & - \\
\hline & IMC1 & 0 & - & - & - \\
\hline & IMC2 & $\square$ & - & - & - \\
\hline & Inertia & 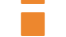 & - & - & - \\
\hline & INN & $\square$ & - & ○ & - \\
\hline & Maximum Probability & $\square$ & - & - & - \\
\hline & Sum Entropy & 0 & - & - & - \\
\hline & Uniformity & $\square$ & - & - & - \\
\hline & Variance $15^{+}$(Global) & & & & \\
\hline & Variance $45^{+}$(Global) & & & & \\
\hline & Variance $45^{-}$(Global) & & & & \\
\hline & Thickness (Quadrants) & - & - & 0 & - \\
\hline \multirow{10}{*}{ INL } & Cluster Prominence & 0 & - & - & - \\
\hline & Correlation & 0 & - & - & - \\
\hline & Maximum Probability & 0 & - & - & - \\
\hline & Variance $15^{+}$(Global) & & & & \\
\hline & Variance $15^{-}$(Global) & & & & \\
\hline & Variance $45^{+}$(Global) & & & & \\
\hline & Variance $45^{-}$(Global) & & & & \\
\hline & Variance $75^{+}$(Global) & & & & \\
\hline & Variance $75^{-}$(Global) & & & & \\
\hline & Thickness (Quadrants) & - & - & 0 & - \\
\hline \multirow{7}{*}{ OPL } & Cluster Prominence & - & - & 0 & - \\
\hline & Cluster Shade & - & - & - & 0 \\
\hline & Entropy & - & - & $\square$ & - \\
\hline & Homogeneity & - & - & O & - \\
\hline & Maximum Probability & - & - & 0 & - \\
\hline & Sum Average & - & - & - & - \\
\hline & Uniformity & - & - & 0 & - \\
\hline \multirow{5}{*}{ ONL } & Cluster Shade & - & - & $\square$ & - \\
\hline & Sum Average & 口 & - & * & - \\
\hline & Sum of Squares & - & - & $\square$ & - \\
\hline & Sum Variance & - & - & 0 & - \\
\hline & Variance $45^{+}$(Global) & & & & \\
\hline
\end{tabular}


Tabela 5 Features showing a moderate $(0.5-0.7)$ and strong $(>0.7$; bold font) Pearson Correlation Coefficient with thickness, per layer, and per quadrant in the case of the local features, for all subjects at study. Q1 - superior-temporal quadrant; Q2 - superior-nasal quadrant; Q3 - inferior-temporal quadrant and Q4- inferior-nasal quadrant. In the features IMC1 and IMC2, IMC stands for Information Measure of Correlation; and INN stands for Inverse Difference Normalized.

\begin{tabular}{|c|c|c|c|c|c|}
\hline Layer & Feature & Q1 & Q2 & Q3 & Q4 \\
\hline RNFL & $\begin{array}{l}\text { Difference Entropy } \\
\text { Homogeneity } \\
\text { IMC1 } \\
\text { IMC2 } \\
\text { INN } \\
\text { Variance } 15^{+} \text {(Global) } \\
\text { Variance } 15^{-} \text {(Global) } \\
\text { Variance } 45^{+} \text {(Global) } \\
\text { Variance } \mathbf{4 5}^{-} \text {(Global) } \\
\text { Variance } 75^{+} \text {(Global) } \\
\text { Variance } 75^{-} \text {(Global) }\end{array}$ & $\begin{array}{l}- \\
- \\
- \\
- \\
-\end{array}$ & $\begin{aligned} &- \\
&- \\
&- \\
&- \\
&- \\
& \\
& \\
& \\
& \\
& \\
& 0 \\
& 0 \\
& 0 \\
& 0 \\
& c\end{aligned}$ & $\begin{array}{l}- \\
- \\
- \\
- \\
- \\
57 \\
58 \\
52 \\
81 \\
58 \\
58\end{array}$ & $\begin{array}{c}-0.51 \\
0.51 \\
-0.52 \\
0.50 \\
0.50\end{array}$ \\
\hline IPL & $\begin{array}{l}\text { Autocorrelation } \\
\text { Sum Variance }\end{array}$ & $\begin{array}{l}- \\
-\end{array}$ & $\begin{array}{l}0.52 \\
0.53\end{array}$ & $\begin{array}{l}- \\
-\end{array}$ & $\begin{array}{l}- \\
-\end{array}$ \\
\hline INL & Variance $45^{-}$(Global) & \multicolumn{4}{|c|}{0.51} \\
\hline OPL & $\begin{array}{l}\text { Variance } 15^{+} \text {(Global) } \\
\text { Variance } 15^{-} \text {(Global) } \\
\text { Variance } 45^{+} \text {(Global) } \\
\text { Variance } 45^{-} \text {(Global) } \\
\text { Variance } 75^{+} \text {(Global) } \\
\text { Variance } 75^{-} \text {(Global) }\end{array}$ & & & $\begin{array}{l}54 \\
54 \\
59 \\
71 \\
56 \\
59\end{array}$ & \\
\hline
\end{tabular}

study demonstrates that texture features do convey information not conveyed by thickness, thus paving the way for their use as biomarkers.

In this study, we performed texture analysis of the MVF image for each of the inner six retinal layers (from the RNFL to the ONL) [13] to identify differences in the retina associated with the subjects' sex. Specifically, we segmented the inner six layers of the retina and computed a fundus image for each. These images were then split into $7 \times 7$ blocks, of which 36 were individually analysed by computing local texture metrics (GLCM). Also, global texture metrics (DTCWT) were computed from the entire fundus image. Finally, the average retinal thickness (for the entire OCT-scanned area of the retina), and the average retinal thickness of each retinal quadrant were computed. A massive distinction between female and male subjects was found when considering the total number of subjects in each group, confirming the findings in a preliminary work [21]. The most relevant differences between groups were found in the IPL and INL, with particular focus on the latter, while the RNFL, the layer on the top of the preferences when it comes to assessing neuroretinal changes, presents only minute differences. When splitting the dataset by age, and performing the same study on sex-related differences, it is notorious that the youngest group conveys most of the information, and very few differences 
between females and males were found in the older group.

It is of particular interest the fact that texture analysis and retinal thickness convey distinct (and eventually complementary) information on the status of the retina, while texture analysis is not very often used when analysing OCT data of the retina. This study demonstrates the potential use of texture, and the level of information conveyed, which fits well to the use of machine learning approaches, as demonstrated in [23] and [5].

The use of both eyes is a limitation of the present study imposed by the limited number of subjects in our database available for the age-match required. This limitation is even further present when assessing the differences for the younger and older groups. Nonetheless, the found differences are clear enough to allow drawing conclusions with confidence.

Further work on this subject will focus on the premenopause, menopause and postmenopause periods to account for the different stages of the female hormonal life cycle.

\section{Conclusion}

The present study sheds light on sex-based differences in the human retina. In this study, we provide evidence suggesting the neuroretina, the visible part of the central nervous system (CNS), presents distinct characteristics for females and males, and that these differences strongly attenuate with age. Second, we demonstrate how texture analysis conveys information not conveyed by the thickness, and that vast differences occur at the different neuroretinal layers. Finally, we demonstrated that texture and thickness are not correlated, for the most part, suggesting texture as a potential biomarker of eye and CNS status in health and disease.

\section{Conflict of interest}

The authors declare that they have no conflict of interest.

\section{Referências}

1. Abràmoff, M.D., Garvin, M.K., Sonka, M.: Retinal imaging and image analysis. IEEE Reviews in Biomedical Engineering 3, 169-208 (2010). DOI 10.1109/RBME.2010. 2084567

2. Adhi, M., Aziz, S., Muhammad, K., Adhi, M.I.: Macular thickness by age and gender in healthy eyes using spectral domain optical coherence tomography. PLoS ONE 7(5), e37638 (2012). DOI 10.1371/journal.pone.0037638 
3. Anantrasirichai, N., Achim, A., Morgan, J.E., Erchova, I., Nicholson, L.: SVM-BASED TEXTURE CLASSIFICATION IN OPTICAL COHERENCE TOMOGRAPHY. 2013 IEEE 10th International Symposium on Biomedical Imaging: From Nano to Macro pp. $1332-1335(2013)$

4. Association, W.M.: Declaration of helsinki - ethical principles for medical research involving human subjects. Journal of the American Medical Association 310(20), 2191-2194 (2013). DOI 10.3917/jib.151.0124

5. Bernardes, R., Jorge, L., Nunes, A., Castelo-Branco, M.: Machine learning approaches in OCT: Application to neurodegenerative disorders, pp. 507-521. Springer International Publishing, Cham (2020). DOI 10.1007/978-3-030-26269-3 23

6. Celik, T., Tjahjadi, T.: Multiscale texture classification using dual-tree complex wavelet transform. Pattern Recognition Letters 30, 331-339 (2009). DOI 10.1016/j.patrec.2008. 10.006

7. Chan, A., Duker, J.S., Ko, T.H., Fujimoto, J.G., Schuman, J.S.: Normal macular thickness measurements in healthy eyes using stratus optical coherence tomography. Archives of Ophthalmology 124(2), 193-198 (2006). DOI 10.1016/j.pestbp.2011.02.012. Investigations

8. Clausi, D.A.: An analysis of co-occurrence texture statistics as a function of grey level quantization. Canadian Journal of Remote Sensing 28(1), 45-62 (2002). DOI 10.5589 $\mathrm{m} 02-004$

9. Conners, R.W., Trivedi, M.M., Harlow, C.A.: Segmentation of a high-resolution urban scene using texture operators ( Sunnyvale, California). Computer Vision, Graphics, \& Image Processing 25(3), 273-310 (1984). DOI 10.1016/0734-189x(84)90197-x

10. Çubuk, M., Kasım, B., Koçluk, Y., Sukgen, E.A.: Effects of age and gender on macular thickness in healthy subjects using spectral optical coherence tomography/scanning laser ophthalmoscopy. International Ophthalmology 38(1), 127-131 (2018). DOI 10. $1007 /$ s10792-016-0432-z

11. Garvin, M.K., Abràmoff, M.D., Wu, X., Russell, S.R., Burns, T.L., Sonka, M.: Automated 3-d intraretinal layer segmentation of macular spectral-domain optical coherence tomography images. IEEE Transactions on Medical Imaging 28, 1436-1447 (2009)

12. Guedes, V., Schuman, J.S., Hertzmark, E., Wollstein, G., Correnti, A., Mancini, R. Lederer, D., Voskanian, S., Velazquez, L., Pakter, H.M., Pedut-kloizman, T., Fujimoto, J.G., Mattox, C.: Optical coherence tomography measurement of macular and nerve fiber layer thickness in normal and glaucomatous human eyes. Ophthalmology 110(1), 177-189 (2003)

13. Guimarães, P., Rodrigues, P., Lobo, C., Leal, S., Figueira, J., Serranho, P., Bernardes, R.: Ocular fundus reference images from optical coherence tomography. Computerized Medical Imaging and Graphics 38, 381-389 (2014). DOI 10.1016/j.compmedimag.2014. 02.003

14. Haralick, R.M.: Statistical and structural approaches to texture. Proceedings of the IEEE 67(5), 786-804 (1979). DOI 10.1109/PROC.1979.11328

15. Haralick, R.M., Shanmugam, K., Dinstein, I.: Textural features for image classification. IEEE Transaction on Systems, Man and Cybernetics SMC-3(6), 610-621 (1973). DOI 10.1109/TSMC.1973.4309314

16. Kashani, A.H., Zimmer-Galler, I.E., Shah, S.M., Dustin, L., Do, D.V., Eliott, D., Haller, J.A., Nguyen, Q.D.: Retinal thickness analysis by race, gender, and age using stratus OCT. American Journal of Ophthalmology 149, 496-502 (2010). DOI 10.1016/j.ajo. 2009.09.025

17. Kassner, A., Thornhill, R.E.: Texture analysis: a review of neurologic MR imaging applications. American Journal of Neuroradiology 31, 809-816 (2010). DOI 10.3174/ ajnr.A2061

18. Kelty, P.J., Payne, J.F., Trivedi, R.H., Kelty, J., Bowie, E.M., Burger, B.M.: Macular thickness assessment in healthy eyes based on ethnicity using stratus OCT optical coherence tomography. Investigative Ophthalmology and Visual Science 49(6), 2668-2672 (2008). DOI 10.1167/iovs.07-1000

19. Li, K., Wu, X., Chen, D.Z., Sonka, M.: Optimal surface segmentation in volumetric images-a graph-theoretic approach. IEEE Transactions on Pattern Analysis and Machine Intelligence 28(1), 119-134 (2006). DOI 10.1109/TPAMI.2006.19 
20. Nunes, A., Ambrósio, A.F., Castelo-Branco, M., Bernardes, R.: Texture biomarkers of Alzheimer's disease and disease progression in the mouse retina. In: IEEE International Conference on Bioinformatics and Bioengineering, pp. 41-46 (2018). DOI 10.1109/ BIBE.2018.00016

21. Nunes, A., Serranho, P., Quental, H., Ambrósio, A.F., Castelo-Branco, M., Bernardes, R.: Sexual dimorphism of the adult human retina assessed by optical coherence tomography. In: J. Henriques, N. Neves, P. de Carvalho (eds.) XV Mediterranean Conference on Medical and Biological Engineering and Computing - MEDICON 2019, pp. 18301834. Springer International Publishing, Cham (2020)

22. Nunes, A., Silva, G., Alves, C., Batista, S., Sousa, L., Castelo-Branco, M., Bernardes, R.: Textural information from the retinal nerve fibre layer in multiple sclerosis*. In: 2019 IEEE 6th Portuguese Meeting on Bioengineering (ENBENG), pp. 1-4 (2019). DOI 10.1109/ENBENG.2019.8692454

23. Nunes, A., Silva, G., Duque, C., Januário, C., Santana, I., Ambrósio, A.F., CasteloBranco, M., Bernardes, R.: Retinal texture biomarkers may help to discriminate between alzheimer's, parkinson's, and healthy controls. PLOS ONE 14(6), 1-13 (2019). DOI 10.1371/journal.pone.0218826

24. Nuzzi, R., Scalabrin, S., Becco, A., Panzica, G.: Gonadal hormones and retinal disorders: A review. Frontiers in Endocrinology 9, 66 (2018). DOI 10.3389/fendo.2018.00066

25. Ooto, S., Hangai, M., Tomidokoro, A., Saito, H., Araie, M., Otani, T., Kishi, S., Matsushita, K., Maeda, N., Shirakashi, M., Abe, H., Ohkubo, S., Sugiyama, K., Iwase, A. Yoshimura, N.: Effects of age, sex, and axial length on the three-dimensional profile of normal macular layer structures. Investigative Ophthalmology and Visual Science 52(12), 8769-8779 (2011). DOI 10.1167/iovs.11-8388

26. Selesnick, I.W., Baraniuk, R.G., Kingsbury, N.C.: The dual-tree complex wavelet transform. IEEE Signal Processing Magazine pp. 123-151 (2005). DOI 10.1109/MSP.2005. 1550194

27. Shaqiri, A., Roinishvili, M., Grzeczkowski, L., Chkonia, E., Pilz, K., Mohr, C., Brand, A., Kunchulia, M., Herzog, M.H.: Sex-related differences in vision are heterogeneous. Scientific Reports 8(7251) (2018). DOI 10.1038/s41598-018-25298-8

28. Soh, L.., Tsatsoulis, C.: Texture analysis of sar sea ice imagery using gray level cooccurrence matrices. IEEE Transactions on Geoscience and Remote Sensing 37(2), 780-795 (1999). DOI 10.1109/36.752194

29. Song, W.K., Lee, S.C., Lee, E.S., Kim, C.Y., Kim, S.S.: Macular thickness variations with sex, age, and axial length in healthy subjects: A spectral domain-optical coherence tomography study. Investigative Ophthalmology and Visual Science 51(8), 3913-3918 (2010). DOI 10.1167/iovs.09-4189

30. Vanston, J.E., Strother, L.: Sex differences in the human visual system. Journal of Neuroscience Research 95, 617-625 (2017). DOI 10.1002/jnr.23895

31. Wang, S., Lu, S., Dong, Z., Yang, J., Yang, M., Zhang, Y.: Dual-tree complex wavelet transform and twin support vector machine for pathological brain detection. Applied Sciences 6(169) (2016). DOI 10.3390/app6060169

32. Wong, A.C., Chan, C.W., Hui, S.P.: Relationship of gender, body mass index, and axial length with central retinal thickness using optical coherence tomography. Eye 19(3), 292-297 (2005). DOI 10.1038/sj.eye.6701466 